\title{
Economics
}

2021; 10(3): 94-104

$\mathrm{http}: / / w w w . s c i e n c e p u b l i s h i n g g r o u p . c o m / \mathrm{j} / \mathrm{eco}$

doi: $10.11648 /$ j.eco.20211003.14

ISSN: 2376-659X (Print); ISSN: 2376-6603 (Online)

\section{An Analytical Expression for Credit Valuation Adjustment Pricing with Wrong-Way Risk}

\author{
Kelin Pan ${ }^{1}$, Chandra Khandrika ${ }^{2}$ \\ ${ }^{1}$ Citibank, Tampa, USA \\ ${ }^{2}$ Citibank, New York, USA
}

Email address:

Kelin.Pan@citi.com (K. Pan), Chandra.Khandrika@citi.com (C. Khandrika)

\section{To cite this article:}

Kelin Pan, Chandra Khandrika. An Analytical Expression for Credit Valuation Adjustment Pricing with Wrong-Way Risk. Economics. Vol. 10, No. 3, 2021, pp. 94-104. doi: 10.11648/j.eco.20211003.14

Received: May 23, 2021; Accepted: June 9, 2021; Published: September 6, 2021

\begin{abstract}
Recently, financial institutions were required to provide the financial derivatives instrument level credit valuation adjustment (CVA) by the new accounting standard. CVA trading desks are facing difficulties to calculate a netting-set level CVA with wrong-way risk (WWR) since the dynamics of the exposures and probability of default (PD) are separated and calculated by different counterparty credit risk (CCR) computing systems. Another difficult work is that the netting-set level CVA mixed the pricing models for all trades under a netting-set. It is significant to develop a new CVA model that is based on the credit adjustment to the existing pricing model under one risk-neutral framework. This paper presents the work on CVA with WWR under the credit deterioration dynamics in both normal and stressed economic conditions. In terms of the doublecorrelation structure that is constructed based on the Gaussian latent variable models we propose an analytical expression of CVA for the fundamental financial derivatives such as futures or forwards contracts. The double-correlation structure captures the market- and asset-credit correlations. The proposed CVA pricing framework is based on the credit deterioration dynamics rather than default dynamics. The credit deterioration index (CDI) is defined as the limit of the credit deterioration variable and calculated using the rating agency credit rating transition data. The proposed CVA with WWR model is a function of the correlations, CDI, counterparty probability of default, loss given default, interest rate and volatility of the traded derivatives. The market- and asset-credit correlation parameters are calibrated to either the normal or stressed market. Under the stressed market, the scenario design, shock variable selection and shock magnitude are discussed. The numerical results show that the CVA is an increasing function of the market-credit correlation and a decreasing function of the credit rating. The stressed CVA is about four times higher than the normal CVA.
\end{abstract}

Keywords: Wrong-Way Risk, Credit Deterioration Dynamics, Market-Credit Correlation, Stressed CVA, Gaussian Latent Variable Models

\section{Introduction}

Counterparty credit risk (CCR) has received a lot of attention from financial institutions since the 2007-2009 Global Financial Crisis (GFC). The credit valuation adjustment (CVA) is the market value of the CCR. The CVA models impact not only capital charge but also pricing of the transactions with counterparties faced by financial institutions. On the other hand, CVA values are required to be reported to the regulators for fair value accounting purpose. It is necessary to develop an accurate and fast computing approach to calculate CVA. As Basel III addressed, the major credit losses during the GFC were from the counterparty credit downgrade or deterioration rather than the actual default. [1] Basel III also addressed CCR under financial and economic stress. Both financial stress and rating downgrade are major concerns by Basel III. The major reforms introduced by Basel Committee on Banking Supervision (BCBS) are (a) "Going forward, banks must determine their capital requirement for counterparty credit risk using stressed inputs". (b) "Banks will be subject to a capital charge for potential mark-to-market losses (ie credit valuation adjustment - CVA - risk) associated with a deterioration in the credit worthiness of a counterparty" (Reference [1], paragraph 14).

Incorporation of all regulatory concerns in a CVA 
calculation is the most important challenge in the pricing and hedging areas for trading desks. CVA captures the changes of the credit and market risk factors as well as their correlations. CVA desks are charged with pricing explicitly for the credit risk in all derivative transactions for financial institutions. CVA pricing for the derivatives instruments is complex due to the asymmetric counterparty credit exposure and incorporation of the counterparty probability of default (PD). It is even more sophisticated to consider the credit downgrade and wrong-way risk (WWR) simultaneously.

Past work has studied CVA with WWR. Redon presented a wrong-way exposure for the normally distributed mark-tomarket (MTM) trade values. [2] Brigo and Pallavicini considered the correlation between default and interest rates in the CDS pricing model. [3] Brigo, Chourdakis and Bakkar analyzed WWR impact on CVA for crude oil swaps. [4] Pykhtin and Rosen allocated the counterparty-level CVA to individual trades and obtained an analytical expression for the expected exposure contributions under an assumption of the normally distributed trade values [5]. Cespedes, Herrero, Rosen and Saunders and Rosen and Saunders developed a simulation algorithm to capture CVA-general WWR and CVA-specific WWR. [6, 7] Hull and White postulated a hazard function that is exponentially related to counterparty exposures. [8] Ghamami and Goldberg proposed a stochastic intensity model to capture the WWR. [9] Lipton and Sepp studied the impact of counterparty asset dynamics on CVA. [10] Pang, Chen and Li derived a correlated exposure for the CVA calculation with WWR. [11] Chung and Gregory calibrated the CVA with WWR model to the quanto credit default swap market. [12] Pan, Pan and Khandrika derived an analytical expression for CVA with WWR using the Gaussian copula model and calculated CVA for commodity futures and equity forwards. [13, 14] It is hard to list all of the studies on CVA with WWR models. The more comprehensive studies on CVA with WWR can be found in the Gregory's books. $[15,16]$

In this paper, we establish a framework to calculate CVA by introducing a conditional loss function based on the Basel III definition. The trade prices for forwards or futures are assumed to be lognormally distributed, which is widely adopted in industry. [17] Then we develop a conditional expected exposure and a conditional PD to calculate CVA with WWR for a given counterparty. The conditional expected exposure is conditional on the default time. Under the risk-neutral probability measure, the expectation is taken under a joint distribution of the market risk factor and the default time. On the other hand, the conditional PD is conditional on the credit deterioration variable. Since the proposed model is based on the credit deterioration dynamics instead of default dynamics, the default time is mapped to the credit deterioration variable. The WWR is specified by the one-factor Gaussian latent variable model that specifies the market-credit correlation. The conditional PD is derived based on another one-factor Gaussian latent variable model that specifies the asset-credit correlation. With certain sophisticated derivations we obtain an analytical expression for CVA with WWR.

There are several new concepts we first propose in order to derive an analytical expression for CVA with WWR. The credit deterioration variable is a key concept under the credit deterioration dynamics. To compute the integral in the CVA formula, the credit deterioration index (CDI) is introduced as the upper limit of the integrand variable, i.e. the credit deterioration variable. The CDI is determined by the credit rating transition matrices published by the rating agencies. The double-correlation structure of the model is an innovation, which captures market- and asset-credit correlations. In the proposed model, the credit deterioration variable dominates the CVA calculation while the counterparty PD is one of the model inputs. Including the credit rating information in a CVA formula is important not only for the computation but also for the Comprehensive Capital Assessment Review (CCAR) CVA reporting since FR Y-14 report requires the CVA data classified by the credit rating. [18]

Two practical approaches are proposed to calibrate the market- and asset-credit correlation parameters to the benchmark values respectively. The proposed model is applied to calculate CVA with WWR under either normal or stressed markets. To calculate the stressed CVA we discuss the process on the scenario design, the variable selection and the shocks to obtain the variables under the stressed market. The stressed CVA is calculated via the shocks on the model parameters under the stressed market environment. We also calculate the CVA loss that is the difference between the stressed CVA and normal one. The CVA loss is a part of the MtM loss of a counterparty for the risk capital calculation. It is also required by FRB to report the difference between the stressed and unstressed aggregated CVA in FR Y-14 report. [18] The numerical results show that the higher the marketcredit correlation, the greater the CVA loss. The numerical results also reveal that the normal CVA, stressed CVA and CVA loss are all increasing functions of the market-credit correlation and decreasing functions of the risk rating.

The rest of the paper is organized as follows. Section 2 establishes a framework for CVA pricing model under credit deterioration dynamics. Section 3 derives the CVA formula under the risk- neutral framework for forwards or futures contracts. Section 4 is dedicated to the numerical examples. Section 5 represents the conclusions. The appendices provide the details of the derivations.

\section{CVA Under Credit Deterioration Dynamics}

Recall the definition of CVA by Basel III: [1] CVA is the risk that associated with a deterioration in the credit worthiness of a counterparty. Basel III also addresses the CVA risk as the one that was a greater source of losses than that arising from outright defaults (reference [1], paragraph 14-(b)). The loss function should reflect the definition of Basel III, that is the loss due to the deterioration in the credit 
worthiness of a counterparty. In fact, the financial institutions will experience the losses from the underlying securities prices down once the securities issuers' credit ratings are downgraded. Similarly, once a counterparty's credit rating is downgraded the financial institutions will have more credit risk that increases the cost of the replacement prior to the counterparty default. In this case, the CVA value is increased to reflect such a credit event. We define such kinds of losses as the conditional losses that are conditional on the credit deterioration events such as credit rating downgrades. The conditional loss function for a credit deterioration at time $\tau$ can be expressed as

$$
L_{t}=1_{\{\tau<T \mid Y=y\}} L_{G D} E(t, X) \mid \tau=t
$$

where $T$ is the maturity of the derivatives transaction, $L_{G D}$ is the loss given default that is equal to one minus recovery rate, $E($.$) is the credit exposure to a financial institution for a$ given counterparty, $0<t<T, X$ represents the market risk factor, $Y$ represents the credit event, and $1_{\{.\}}$is the indicator function that takes value one if the argument is true and zero otherwise. For CCR we take the positive value of the markto-market (MtM) value of a derivative transaction as the credit exposure of a counterparty. Denoting the positive MtM value of a transaction as $V^{+}(t, X)$ we have $E(t, X)=V^{+}(t, X)$. For simplicity, we also consider the loss given default as constant. Like other pricing models, CVA pricing is also under the risk-neutral probability measure. Taking expectation of the discounted loss function (1) under the risk-neutral probability measure $Q$ (it is the market convention to employ the money market account as the numeraire and denoted by $Q$ ) and integrating from the current time at $t=0$ to the maturity time $T$, we have

$$
C V A=L_{G D} \int_{0}^{T} E E^{Q}(t, X \mid \tau=t) d P D(t \mid Y=y)
$$

where

$$
E E^{Q}(t, X \mid \tau=t)=E^{Q}\left[D_{0, t} E(t, X) \mid \tau=t\right]=E^{Q}\left[D_{0, t} V^{+}(t, X) \mid \tau=t\right]
$$

whereas $D_{0, t}$ is the discount factor. Under the credit deterioration dynamics we need to map the default time $\tau$ to the credit deterioration variable $Y$, which keeps consistent with the expression of the conditional PD. The mapping is unique. [19] The mapping changes the joint distribution of $(\tau, X)$ to that of $(Y, X)$. The interval of the integral in (2) has been changed from $[0, T]$ to $\left(-\infty, y_{s}\right)$, where $y_{s}$ is CDI as a model input parameter and will be discussed later. To keep consistent with the pricing models for the same products we take $V^{+}(T, X)$ as the payoff function of the traded product. Thus under the credit deterioration dynamics, the expected exposure (3) can be written as

$$
E E^{Q}(T, X \mid Y=y)=E^{Q}\left[D_{0, T} V^{+}(T, X) \mid Y=y\right]
$$

which is a function of $y$. And CVA in (2) can be rewritten as

$$
C V A=L_{G D} \int_{-\infty}^{y_{s}} E E^{Q}(T, X \mid Y=y) d P D(T \mid Y=y)
$$

Equation (5) is obtained under the credit deterioration dynamics. Once the payoff function $V^{+}(T, X)$ and conditional $\mathrm{PD} P D(t \mid Y=y)$ are determined the integral in (5) is easily computed by either numerical computing technique such as the trapezoid method or an analytical formula if the trade price has a simple payoff function with a simple conditional PD. The most advantage using the credit deterioration dynamics is to avoid thousands of simulation paths since the default paths have been replaced by a distribution of the credit deterioration random variable. Therefore, equation (5) provides a fast way to compute CVA for each trade, even combining the WWR. With WWR the market risk factor $X$ is associated with the credit deterioration variable $Y$. We will discuss the CVA with WWR model in the next section.

\section{CVA with WWR}

We consider a forwards or futures contract, whose price process follows the following lognormal dynamics (reference [17], equation (6.1) on page 198)

$$
d V(t, X)=\sigma_{t} V(t, X) d W_{t}^{Q}
$$

where $\sigma_{t}$ is the instantaneous volatility of the forwards or futures price and $W_{t}^{Q}$ is the Wiener process under the risk neutral probability measure $Q$. The solution of (6) can be obtained using Itô's Lemma

$$
V(T, X)=V_{0} e^{-\frac{1}{2} \int_{0}^{T} \sigma_{u}^{2} d u+\int_{0}^{T} \sigma_{u} d W_{u}^{Q}}
$$

where $V_{0}=V(0, X(0))$. For CVA pricing, the WWR has more significant impact on CVA than that from the volatility. Under the risk neutral probability measure, a constant implied volatility is assumed. In this case the forwards price is simplified as

$$
V(T, X)=V_{0} e^{-\frac{1}{2} \sigma^{2} T+Z \sigma \sqrt{T}}
$$

where $W_{T}^{Q}-W_{0}^{Q}=Z \sqrt{T}, Z$ is the standard normal random variable and represents the market risk factor. The WWR is specified by the following one-factor Gaussian latent variable model [20]

$$
Z=\rho Y+\omega \sqrt{1-\rho^{2}}
$$

where $\rho$ is the correlation parameter between $Z$ and $Y, Y$ and $\omega$ are standard normal random variables and independent of each other. $\rho$ represents the market-credit correlation. The random variable $Y$ represents the systematic risk factor for the 
credit deterioration in an economy or financial system. The random variable $\omega$ represents the idiosyncratic market risk factor. Both $Y$ and $\omega$ impact on the market risk factor in a different way. When $\rho$ is close to one the market risk factor is mostly caused by $Y$. Since $Y$ is the systematic risk factor, all counterparties in the system will experience a similar credit deterioration. An example is that, during the GFC period, a massive CDS contracts were unwound and all counterparties

$$
E E^{Q}(T \mid Y=y)=E^{Q}\left[\left(D_{0, T} V(T, X)\right)^{+} \mid Y=y\right]=D_{0, T} V_{0} E^{Q}\left[e^{-\frac{1}{2} \sigma^{2} T+\sigma \sqrt{T}\left(\rho y+\omega \sqrt{1-\rho^{2}}\right)}\right]
$$

where $(.)^{+}=\max (., 0)$. As discussed in the last section, under the credit deterioration dynamics, the default time variable $\tau$ has been mapped to the normal random variable $Y$ since $Y$ has been defined as the credit deterioration variable. The mapping changes the default dynamics to the

$$
E E^{Q}(T \mid Y=y)=D_{0, T} V_{0} \int_{-\infty}^{\infty}\left(e^{-\frac{1}{2} \sigma^{2} T+\sigma \sqrt{T}\left(\rho y+\omega \sqrt{1-\rho^{2}}\right)}\right) \phi(y) \phi(\omega) d \omega
$$

where $\phi($.$) is the standard normal probability density$ function. For a standard normal random variable $\chi$ its probability density function is

$$
\phi(\chi)=\frac{1}{\sqrt{2 \pi}} e^{-\chi^{2} / 2}
$$

Denoting

$$
g(\omega)=e^{\omega \sigma \sqrt{T} \sqrt{1-\rho^{2}}} \phi(\omega)
$$

and considering the result in Appendix I, $g(\omega)$ can be

$$
E E^{Q}(T \mid Y=y)=V_{0} D_{0, T} e^{y \rho \sigma \sqrt{T}-\rho^{2} \sigma^{2} T / 2} \phi(y) \int_{-\infty}^{\infty} \phi(u) d u=V_{0} D_{0, T} e^{y \rho \sigma \sqrt{T}-\rho^{2} \sigma^{2} T / 2} \phi(y)
$$

Substituting (12) into (5) we obtain the expression of CVA with WWR

$$
C V A=L_{G D} V_{0} D_{0, T} \int_{-\infty}^{y_{s}} e^{y \rho \sigma \sqrt{T}-\rho^{2} \sigma^{2} T / 2} \phi(y) d P D(T \mid Y=y)
$$

The conditional PD could be complicated in general. However, for a large homogeneous portfolio a well-known conditional PD has been developed by Vasicek and widely used in industry. [20, 21] To obtain such a conditional PD we assume that the counterparty asset follows another Gaussian latent variable model

$$
A_{t}=\beta Y+\varepsilon_{t} \sqrt{1-\beta^{2}}
$$

where $Y$ is the systematic risk factor defined by (8), $\varepsilon_{t}$ is the individual specific factor for a given counterparty and $\beta$ is the correlation between the counterparty asset and systematic risk factor. The variables $Y$ and $\varepsilon_{t}$ are standard normal random variables and independent of each other. Though a normal random variable could go to infinity, in the real world expressed as

$$
g(u)=\phi(u) e^{\frac{1}{2} \sigma^{2} T\left(1-\rho^{2}\right)}
$$

where

$$
u=\omega-\sigma \sqrt{T} \sqrt{1-\rho^{2}}
$$

Substituting $g(u)$ into the integral of $E E^{Q}(T \mid Y=y)$ in (10) and replacing the integrand variable $\omega$ by $u$ yield

there exists a limit of the variable. We denote the limit as CDI that is given in the next section. The impact of the counterparty asset on CVA has been studied by Lipton and Sepp. [10] Conditional on the systematic risk factor $Y$, a well-known conditional PD is given by [21]

$$
P D(t \mid Y=y)=\Phi\left(\frac{C_{t}-\beta y}{\sqrt{1-\beta^{2}}}\right)
$$

where $\Phi($.$) is standard normal cumulative density function$ and $C_{t}$ is the threshold of the counterparty asset price. The counterparty will default once $A_{t}<C_{t}$. The parameter $C_{t}$ is determined by the individual counterparty PD

$$
C_{t}=\Phi^{-1}\left(P D_{t}\right)
$$

where $\Phi^{-1}($.$) is the inverse of the standard normal$ cumulative density function, $P D$ is the unconditional probability of default for a given counterparty, which is given as a model input. Since (16) is obtained under an assumption of a large homogeneous portfolio we assume that the 
parameter $C_{t}$ is constant as if all counterparties in the portfolio have same credit qualities like a single counterparty. For an inhomogeneous portfolio, $C_{t}$ is no longer a constant. Generally speaking, the conditional PD of (15) is not applicable in this case. However, if (15) is held for each individual counterparty, the portfolio CVA can be obtained by integrating the individual CVA with respect to $C_{t}$. In this paper, a homogeneous portfolio is assumed and $C_{t}$ is considered as constant (i.e. $C_{t}=C$ ). The differentiation of the conditional PD with respect to $y$ yields

$$
d P D(t \mid Y=y)=\phi\left(\frac{C-\beta y}{\sqrt{1-\beta^{2}}}\right)\left(-\frac{\beta}{\sqrt{1-\beta^{2}}}\right) d y
$$

We propose a practical approach to estimate $\beta$ using the negative value of the correlation between the counterparty's stock price and the economic growth, based on an assumption that the credit deterioration variable and the economic growth both are normally distributed but with opposite economic meaning. This idea will be further illustrated using a numerical example in the next section. Substituting (17) into (13) yields

$$
C V A=-\frac{\beta L_{G D} V_{0} D_{0, T}}{\sqrt{1-\beta^{2}}} \int_{-\infty}^{y_{s}} e^{y \rho \sigma \sqrt{T}-\rho^{2} \sigma^{2} T / 2} \phi\left(\frac{C-\beta y}{\sqrt{1-\beta^{2}}}\right) \phi(y) d y
$$

where $y_{s}$ is CDI that reflects the counterparties' credit downgrade in an economy or financial system. It is determined by the rating transition matrices given by the rating agency. For example, the CDI from an original rating $A A A$ downgraded to $B B$ is 3.15 (Table 3 below). In (18) the multi-time step scenarios have been extended to the single-time step scenarios, which implies that the variable $Y$ does not depend on time $t$. This assumption is similar to the Single Scenario Ordering method proposed by Rosen and Saunders. [7] Under the multi-time step scenarios Rosen and Saunders and Brigo, [7] Chourdakis and Bakkar [4] gave the CVA expression in a discrete time.

Denoting

$$
h(y)=e^{y \rho \sigma \sqrt{T}} \phi\left(\frac{C-\beta y}{\sqrt{1-\beta^{2}}}\right) \phi(y)
$$

and considering the results in Appendix II, the above equation can be expressed as

$$
h(v)=\phi(v) \phi(A) e^{\frac{1}{2} \rho^{2} \sigma^{2} T}
$$

where

$$
\begin{gathered}
v=\frac{y}{\sqrt{1-\beta^{2}}}-A_{1} \\
A=C-\beta \rho \sigma \sqrt{T} \\
A_{1}=\frac{\beta C}{\sqrt{1-\beta^{2}}}+\rho \sigma \sqrt{T} \sqrt{1-\beta^{2}}
\end{gathered}
$$

The variable $y$ has been replaced by a new variable $v$. Substituting (20) into (18) yields

$$
C V A=-\beta L_{G D} V_{0} D_{0, T} \phi(A) \int_{-\infty}^{v_{s}} \phi(v) d y=-\beta L_{G D} V_{0} D_{0, T} \phi(A) \Phi\left(v_{s}\right)
$$

where

$$
v_{s}=\frac{y_{s}}{\sqrt{1-\beta^{2}}}-A_{1}
$$

Since the asset price will decrease when the credit deterioration variable increases, the correlation between the counterparty asset price and credit deterioration variable should be negative. We can rewrite (24) using an absolute value of $\beta$ for convenience

$$
C V A=|\beta| L_{G D} V_{0} D_{0, T} \phi(A) \Phi\left(v_{s}\right)
$$

Now we obtain an analytical expression for CVA with WWR. Equation (26) has the following characteristics.

1. $\rho=0$ : It is the case without market-credit correlation.

$$
\text { In this case, } A=C, A_{1}=\frac{\beta C}{\sqrt{1-\beta^{2}}} \text { and } v_{s}=\frac{y_{s}-\beta C}{\sqrt{1-\beta^{2}}}
$$

according to (22), (23) and (25). At $\rho=0$, equation
(26) is reduced to

$$
C V A=|\beta| L_{G D} V_{0} D_{0, T} \phi(C) \Phi\left(v_{s}\right)
$$

2. $\rho=1$ : It is the case with maximum market-credit correlation. In this case, $A=C-\beta \sigma \sqrt{T}$, $A_{1}=\frac{\beta C}{\sqrt{1-\beta^{2}}}+\sigma \sqrt{T} \sqrt{1-\beta^{2}}$ and $v_{s}=\frac{y_{s}-\beta C}{\sqrt{1-\beta^{2}}}-\sigma \sqrt{T} \sqrt{1-\beta^{2}}$ according to (22), (23) and (25). CVA is calculated by (26). We can compare the two CVA values at $\rho=0$ and $\rho=1$ while the other parameters are fixed. Due to a bell shape of the normal probability density function, we have $\phi(C-\beta \sigma \sqrt{T})>\phi(C)$. Similarly, the normal cumulative density function is an increasing function of the argument. Thus, we conclude that the CVA at $\rho=1$ is greater than that at $\rho=0$, which indicates that the CVA is an increasing function of $\rho$. 
3. Inhomogeneous Portfolio: As we stated, the CVA formula in (26) is derived under the assumption of a large homogeneous portfolio. For an inhomogeneous portfolio with a large number of counterparties, the parameter $C$ is no longer constant and can be considered as a random number. The portfolio level CVA is obtained by integrating the individual CVA with respect to the variable $C$

$$
C V A^{\text {port }}=|\beta| L_{G D} V_{0} D_{0, T} \int_{-\infty}^{C_{m}} \phi(C) \Phi\left(v_{s}(C)\right) d C
$$

where $C_{m}$ is the maximum value of the counterparty asset price thresholds. We already showed at the first bullet that at $\rho=0$

$$
v_{s}(C)=\frac{y_{s}-\beta C}{\sqrt{1-\beta^{2}}}
$$

In this case, the portfolio CVA can be expressed as

$$
C V A^{\text {port }}=|\beta| L_{G D} V_{0} D_{0, T} \int_{-\infty}^{C_{m}} \phi(C) \Phi\left(\frac{y_{s}-\beta C}{\sqrt{1-\beta^{2}}}\right) d C
$$

Employing the expression of the bivariate normal distribution the portfolio CVA can be expressed as [22]

$$
C A V^{\text {port }}=|\beta| L_{G D} V_{0} D_{0, T} \Phi_{2}\left(y_{s}, C_{m} ; \beta\right)
$$

where $\Phi_{2}($.$) is the bivariate normal cumulative density$ function. The portfolio CVA can be considered as the credit adjusted valuation formula at a portfolio level. In general case, when the parameter $\rho$ is not equal to zero, it is hard to obtain an analytical expression. The numerical integration methods could be employed to calculate the CVA at a portfolio level.

\section{Numerical Examples}

Due to the lack of detailed counterparty information, we consider one of the Exchange listed top 500 companies as a counterparty. The S\&P 500 Futures is a stock market index futures contract traded on the Exchange. It is based on the S\&P 500 stock market index. Its current market price is considered as the current futures contract value for a counterparty. For example, the settlement price of the S\&P 500 Futures is $\$ 4,127.70$ on April 22, 2021 (https://m.investing.com). We also consider the volatility index VIX as a proxy of the implied volatility of the futures. Its market value is 18.71 on April 22, 2021 (finance.yahoo.com/quote/\%5EVIX). The Libor rate is still commonly used by financial institutions to calculate the discount factors. For example, the one year Libor rate is 0.29\% on April 20, 2021 (https://www.global-rates.com). Those market data are available in the public data sources.

The market data given in above paragraph can be employed for CVA calculation. However, the credit data, which includes the counterparty PD, CDI, loss given default and correlations, are not available in market. We will use historical data to estimate the parameters that related to the counterparty credit qualities. First the historical global corporate default rates are employed to estimate the counterparty PD. [23] The following table gives the current (at study time) and historical default rates based on S\&P Global Ratings report (reference [23], Table 1, pages 2-3, not in this paper)

Table 1. Corporate Default Rate (DR) Historical Data.

\begin{tabular}{llll}
\hline Time & DR (\%) & IG-DR (\%) & SG-DR (\%) \\
\hline 2009 & 4.19 & 0.33 & 9.95 \\
2019 & 1.30 & 0.06 & 2.54 \\
2020 & 2.74 & 0.00 & 5.50 \\
\hline
\end{tabular}

source: ref. 23, Table 1. DR: global corporate default rate;

IG/SG-DR: investment/speculative grade default rate.

The table shows during the Global Financial Crisis (GFC) in 2009 , the corporate default rate is almost double what it was in 2020. Due to the impact of the Coronavirus Crisis the default rate in 2020 is about twice the amount it was in 2019. In order to avoid the effect of the Coronavirus Crisis, we consider the default rate in 2019 as the one under the normal market, which is $1.30 \%$ according to Table 1 . It is obvious that the major contribution of the defaults is from the speculative grade default rates. It seems that the crises have less impact on the investment grade fault rates. We will discuss which stressed market will be considered for the stressed CVA calculation below. Once we have default rate that is considered as the individual PD, we can calculate the parameter $C$ by (16). Taking default rate in 2019, we have $C=\Phi^{-1}(1.30 \%)$.

Next, we discuss how to estimate CDI based on the S\&P Global Ratings report. [23] The report studied the long-term historical data from 1981 to 2020 and analyzed the credit rating transition matrices. The rating transitions reflect the historical average that was computed over the multiple static pools. We take one- and three-year Global Corporate Average Transition Rates from AAA or AA rating grade to AAA, $\mathrm{AA}, \mathrm{A}, \mathrm{BBB}, \mathrm{BB}, \mathrm{B}, \mathrm{CCC} / \mathrm{C}, \mathrm{D}\}$ (reference [23], Table 21, page 51-not in this paper) as example to show how to calculate CDI. We only consider the transitions with the available ratings and remove the non-rating (NR) category. For this purpose, we need to rescale the transition matrices without NR category. The rescaled transition matrices are shown in Table 2. After rescaling, the sum of all transition rates in each column should be $100 \%$. The index CDI represents the credit deterioration or credit rating downgrade, which can be calculated using the rating transition matrix. Denoting the CDI as $Y$ and transition rate from state $i$ to state $j$ as $q_{i j}$, the relationship between $q_{i j}$ and the cumulative density function of $Y$ is

$$
q_{i j}=\Phi\left(Y_{i, j}\right)-\Phi\left(Y_{i, j-1}\right)
$$

assuming $\Phi\left(Y_{i, 0}\right)=0$ and $i=1,2$ (corresponding to AAA and 
AA), $j=1, \ldots, 8$ (corresponding to AAA,..., D). The CDI $Y_{s}$ is solved using the iterative approach. The re-scaled transition matrices for one-year and three-year global corporate average rating transition rates are shown in Table 2. For other rating grades, a similar approach can be adopted. The computed CDIs are shown in Table 3.

Table 2. Re-scaled Transition Matrices (\%).

\begin{tabular}{lllll}
\hline Rating & One-year & \multicolumn{3}{c}{ Three-year } \\
\hline to/from & AAA & AA & AAA & AA \\
\hline AAA & 89.85 & 0.50 & 72.02 & 1.27 \\
AA & 9.35 & 90.77 & 24.42 & 75.38 \\
A & 0.55 & 8.09 & 2.56 & 20.38 \\
BBB & 0.05 & 0.49 & 0.35 & 2.19 \\
BB & 0.11 & 0.05 & 0.29 & 0.37 \\
B & 0.03 & 0.06 & 0.09 & 0.24 \\
CCC/C & 0.05 & 0.02 & 0.12 & 0.03 \\
D & 0.00 & 0.02 & 0.14 & 0.13 \\
\hline
\end{tabular}

source: reference [23], Table 21 (not in this paper).

Table 3. Index Ys for AAA and AA Obligors.

\begin{tabular}{lllll}
\hline Rating & One-year & \multicolumn{3}{l}{ Three-year } \\
\hline to/from & AAA & AA & AAA & AA \\
\hline AAA & 1.27 & -2.58 & 0.58 & -2.24 \\
AA & 2.41 & 1.36 & 1.81 & 0.73 \\
A & 2.81 & 2.49 & 2.33 & 1.89 \\
BBB & 2.88 & 2.96 & 2.49 & 2.42 \\
BB & 3.15 & 3.08 & 2.69 & 2.65 \\
B & 3.28 & 3.34 & 2.79 & 2.93 \\
CCC/C & $>3.28$ & 3.53 & 2.98 & 3.00 \\
D & $>3.28$ & $>3.53$ & $>2.98$ & $>3.00$ \\
\hline
\end{tabular}

The positive values in Table 3 represent the CDIs for downgrading while the negative values represent the CDIs for upgrading. For example, a counterparty from an original AAA rating grade to $\mathrm{BB}$ rating grade has $\mathrm{CDI}$ equal to 3.15 (the second column, Table 3 ). Thus, we have $y_{s}=3.15$.

The Loss Given Default for a counterparty with a BB rating is $53 \%$ as reported by Moody's research article (Moody's Investors Service, "Probability of Default Ratings and Loss Given Default Assessments for Non-Financial Speculative-Grade Corporate Obligors in the United States and Canada", August 2006, the table on page 7. Available at https://care-mendoza.nd.ed/asset/152347/loss-given-defaultrating-methodology.pdf).

Now we discuss the approaches for estimation of the correlation parameters $\rho$ and $\beta$. We will discuss how to calibrate the market-credit correlation parameter $\rho$ to either normal or stressed market later. First, we propose an approach to estimate the asset-credit correlation parameter $\beta$ based on the following two assumptions: (1) the counterparty asset is valued by its stock price on the market, (2) the credit deterioration variable and economic growth are both normally distributed but with opposite directions. Since we consider the counterparty on the list of the Exchanges, the counterparty's asset price can be estimated by S\&P 500 Index (available https://finance.yahoo.com/quote/\%5EGSPC/history? $p=\% 5 E$ $G S P C$ ). The economic growth is measured by GDP per capita in USD (available at https://www.macrotrends.net /countries/USA/united-states/gdp-per-capita). The GDP per capita is given annually while the S\&P 500 Index is given monthly. The latter needs to be calculated by average in year. There are 60 data points in total from 1960 to 2019 . Then the Pearson correlation coefficient can be used to find the correlation between the GDP per capita and S\&P 500 Index, which is $93.99 \%$. According to the second assumption, we have $\beta=-93.99 \%$.

The calculation of CVA under a normal market is straightforward. The published CVA values are mostly in normal market environments. We can calibrate the marketcredit correlation directly to those values, which will be illustrated by the examples below. The calculation of CVA under a stressed market is not straightforward. First, we need to define the stress scenarios in a stressed market. Then we need to find which parameters and how much are shocked under the stress scenarios. Then the stressed CVA can be calculated using the parameters selected under the stress scenarios. The above discussions are summarized as follows.

\section{(1) Step 1. Stress Scenario Design}

There are two approaches to design a stress scenario. The first approach is to employ a hypothetical stress scenario such as the CCAR supervisory adverse scenario or supervisory severely adverse scenario. The second approach is to employ an actual stressed market scenario, which is the historical worst economic or financial conditions in the most recent years. We adopt the second approach for the stress scenario design and select the GFC as the stressed market scenario.

(2) Step 2. Parameter Selection for Shock

The fundamental requirement for the selected parameters for shock is that they must be market observable under the designed stressed market. As shown in (26), the proposed CVA model includes eight key parameters: (1) CDI $y_{s}$, (2) implied volatility $\sigma$, (3) threshold $C$, (4) maturity $T$, (5) market-credit correlation $\rho$, (6) asset-credit correlation $\beta$, (7) loss given default and (8) interest rate. The parameter $T$ is constant and given by a contract. The parameters $\rho$ and $\beta$ have no market data under the designed stressed market scenario. Since $y_{s}$ is determined by the rating agency transition matrices and it was not updated during the GFC, the parameter $y_{s}$ also cannot be selected for shock. On the other hand, we consider the loss given default and interest rate as constant and no change during the GFC. However, the market data shows that VIX experienced a maximum value during the GFC in November 20, 2008 (available at https://www.macrotrends.net/2603/vix-volatility-index-

historical-chart), which is 0.8086 . Another parameter to be shocked is the counterparty's PD that determines the parameter $C$.

(3) Step 3. Determination of the Shock Magnitude

We already considered the GFC as the designed stressed market scenario. Based on the market data, the implied volatility is 0.1871 in the normal market and 0.8086 in the stressed market, which indicates a shock magnitude 0.6215 from the normal to stressed market. Table 1 shows the default 
rate is $1.30 \%$ in the normal market (2019) and $4.19 \%$ in the stressed market (2009). It is shocked by $2.89 \%$ from the normal to stressed market.

Except for the individual PD and implied volatility, the other parameters are same for the calculation of either normal or stressed CVA. The parameters used for the numerical computation by (26) are summarized as follows:

a. Credit deterioration index: $y_{s}=3.15$

b. Maturity of the contract: $T=1$ (year)

c. Implied volatility: $\sigma^{n}=18.71 \% \quad$ (normal), $\sigma^{s}=80.86 \%$ (stressed)

d. Current S\&P 500 Futures: $V_{0}=4,127.70$ (USD)

e. Interest rate: $r=0.29 \%$ f. Asset-credit correlation: $\beta=-93.99 \%$

g. Counterparty default rate: $P D^{n}=1.30 \%$ (normal), $P D^{S}=4.19 \%$ (stressed)

h. Loss given default: $L_{G D}=53 \%$

Both the normal and stressed CVAs are calculated by (26) using the data provided above. The numerical results are shown in Table 4 for $C V A^{n}$ under the normal market (Normal), $C V A^{S}$ under the stressed market (Stressed), CVA loss $C V A^{L}=C V A^{S}-C V A^{n} \quad$ (Loss), and CVA ratio $C V A^{R}=C V A^{s} / C V A^{n}$ (Ratio) against the parameter $\rho$.

Table 4. CVA/VO Against the Market-Credit Correlation (in \%).

\begin{tabular}{|c|c|c|c|c|c|c|c|c|c|c|c|}
\hline CVA/ $\rho$ & 0.0 & 0.1 & 0.2 & 0.3 & 0.4 & 0.5 & 0.6 & 0.7 & 0.8 & 0.9 & 1.0 \\
\hline Normal & 1.66 & 1.73 & 1.80 & 1.87 & 1.94 & 2.01 & 2.09 & 2.17 & 2.25 & 2.33 & 2.42 \\
\hline Stressed & 4.44 & 5.05 & 5.71 & 6.42 & 7.18 & 7.98 & 8.81 & 9.68 & 10.57 & 11.48 & 12.39 \\
\hline Loss & 2.78 & 3.33 & 3.92 & 4.56 & 5.24 & 5.96 & 6.72 & 7.51 & 8.32 & 9.14 & 9.97 \\
\hline Ratio & 2.68 & 2.93 & 3.18 & 3.44 & 3.70 & 3.96 & 4.22 & 4.46 & 4.70 & 4.92 & 5.12 \\
\hline
\end{tabular}

note: CVA Ratio is in absolute values.

Once obtained the CVA values we can calibrate the market-credit correlation parameter $\rho$ to either the normal or stressed benchmark value. We first discuss how to calibrate such a parameter to a benchmark value in the normal market using the following example.

The example is based on the model from Brigo, Chourdakis and Bakkar. [4] The authors calculated CVA with WWR for the crude oil forward swaps. The Table 5 (no in this paper) in their paper shows a relative CVA of $1.90 \%$ (i.e. CVA/Notional $=130.39 / 6852.35)$ with an intensity volatility of 0.295 and average correlation of $68.9 \%$. We calibrate $\rho$ to

$C V A^{n} / V_{0}=1.90 \%$ in Table 4, which is between $C V A^{n} / V_{0}=1.87 \%$ and $C V A^{n} / V_{0}=1.94 \%$. Using the interpolation approach, we obtain $\rho=0.343$. The crude oil forward swaps have higher CVA than that for the currency or equity forwards. Therefore, the correlation parameter could be lower than that for the crude oil forward swaps.

Next we discuss how to calibrate the market-credit correlation parameter to the stressed market. First we map our calculated CVA into a credit default swap (CDS) CVA. The CDS CVA is proportional to the CDS spread in the following way (Reference [15], page 172, Equation (7.2))

$$
\frac{C V A}{V^{\text {premium }}}=S^{C D S}
$$

where $V^{\text {premium }}$ is the present value of the premium leg and $S^{C D S}$ is the CDS spread. During the GFC that is the stressed market, a 5-year CDS spread of Lehman Brothers was jumped to 610 basis points on 09/10/2008 Wednesday (source: Reuters, Lehman credit spreads soar to record after loss," Financial Services \& Real Estate, September 10, 2008, by Walden Siew. Available at https://www.reuters.com/article/us-lehman-cds-rbc-
idUSN1040517120080910). If we consider $V_{0}$ in (26) as the equivalent value of $V^{\text {premium }}$ we can set the $C V A^{s} / V_{0}$ in Table 4 to equal to $6.10 \%$. Using the interpolation approach we obtain the implied market-credit correlation parameter $\rho=0.255$. In this way, we calibrated the market-credit correlation parameter to the CDS spread jump under the actual stressed market.

We also calculated the CVA loss and CVA ratio as shown in the last two rows of Table 4. The CVA loss measures the absolute loss in a stressed market. The CVA ratio measures the relative loss in a stressed market. The CVA loss and jump-to-default loss are the MTM losses of the CCR for bank stress testing and risk capital calculation under a stressed financial or economic environment. Our numerical results show that the CVA loss depends on the WWR. For example, the CVA loss is quite small at $\rho=0$, which is $2.78 \%$ of $C V A / V_{0}$ according to Table 4. The CVA loss is quickly increased to $9.97 \%$ of $C V A / V_{0}$ as it reaches to the maximum $\rho$. However, the increasing speed is slow as the marketcredit correlation increases. The CVA ratio gives a relative value of the stressed CVA relative to the normal CVA. Table 4 shows that the CVA ratio increases from 2.68 to 5.12 as the correlation is from 0 to 1 . The average CVA ratio is about 4 , which indicates that the stressed CVA is about four times of the normal one.

\section{Conclusions}

Basel III has emphasized the credit losses caused by the counterparty credit deterioration. According to the definition of CVA by Basel III, we defined a conditional loss function for a counterparty due to a credit deterioration event. Taking expectation for the loss function and changing the default 
dynamics to the credit deterioration dynamics, we obtained an analytical expression for CVA with WWR. The fundamental framework of the proposed model is based on the credit deterioration dynamics for CVA with WWR. The model parameters have been estimated based on either the market data or the historical data. The calibration methodologies have been developed to determine the nonmarket observable parameters. The proposed model is easy not only for CVA computation but also for parameter calibration under both normal and stressed markets.

Basel III also addresses the stress inputs for CCR models. The non-market observable parameters in CCR models are hard to be stressed due to lack of the available data under stressed markets. The corresponding MTM losses caused by those parameters are difficult to be measured. The calibration approaches under the normal market may not work appropriately under the stressed market. The model parameters need to be re-calibrated in this case. We proposed a two-step approach to calibrate the non-market observable parameters to the stressed market. The first step is to calculate stressed CVA with the parameter shocks in a designed stressed market. The second step is to calibrate the non-market observable parameters to the stressed CVA values.

To calculate CVA with WWR, a joint distribution of either the market-default or market-credit random variables is needed. We adopted the credit deterioration dynamics and introduced a credit deterioration variable to link the market and credit risk factors. The model captures the sophisticated correlation structures between the market movements and credit changes through a double-correlation structure. Another new concept employed in this paper is CDI, the credit deterioration index. It specifies the counterparties' credit quality in an economy rather than an individual counterparty's.

The proposed model has the following characteristics: (1) It considers the counterparty's credit quality and default separately; (2) It captures two different correlation structures, i.e. the exposure-credit correlation and asset-credit correlation; (3) The model framework can be used for futures and forwards with various underlying products. The characteristics (1) and (2) give a flexibility of the model to cover the counterparties with different entities. In this case (the counterparty is not same as the legal entity), the systematic credit deterioration variable is shared by both counterparties and legal entities. The credit dependency of the asset price can be employed to describe the credit quality of the underlying reference. The model could also cover a specific WWR if we consider that the exposure is correlated with an individual rating downgrade. The detailed discussion on the specific WWR and general WWR can be found in our previous paper. [14]

The proposed model can be easily extended to more complex products such as CDS and IRS etc. It is convenient and transparent to calculate CVA with WWR using an analytical or a semi-analytical expression. The proposed model can be a reference for both regulators and practitioners. Further work could be conducted by considering the impact of the stochastic recovery, volatility and interest rates as well as the inhomogeneous portfolios in the CVA calculation. Currently we focus on the vanilla products. The future work will be extended to the more complex products.

\section{Acknowledgements}

The first author thanks his colleagues for discussion on this topic. The authors thank the reviewer for his instructive comments. The opinions presented in this paper are only those of the authors and do not represent those of the bank. The authors alone are responsible for the content and the writing of the paper.

\section{Appendix}

\section{Appendix I. Proof of $g(\omega)$ in Equation (10)}

For the standard normal distribution, the probability density function has an exponential form. The power of two exponential functions is equal to the sum of the two powers. Thus, the power of the function $g(\omega)$ in Equation (10) can be expressed as

$$
\begin{aligned}
& \omega \sigma \sqrt{T} \sqrt{1-\rho^{2}}-\frac{1}{2} \omega^{2}=-\frac{1}{2}\left(\omega^{2}-2 \omega \sigma \sqrt{T} \sqrt{1-\rho^{2}}\right) \\
& =-\frac{1}{2}\left[\left(\omega-\sigma \sqrt{T} \sqrt{1-\rho^{2}}\right)^{2}-\sigma^{2} T\left(1-\rho^{2}\right)\right] \\
& =-\frac{1}{2} u^{2}+\frac{1}{2} \sigma^{2} T\left(1-\rho^{2}\right)
\end{aligned}
$$

where $u=\omega-\sigma \sqrt{T} \sqrt{1-\rho^{2}}$. Thus, $g(\omega)$ can be expressed by $g(u)=\phi(u) e^{\frac{1}{2} \sigma^{2} T\left(1-\rho^{2}\right)}$.

\section{Appendix II. Proof of $h(y)$ in Equation (19)}

By means of the definition of the standard normal probability density function, the function $h(y)$ in Equation (19) can be 
expressed as

$$
h(y)=e^{y \rho \sigma \sqrt{T}} \phi\left(\frac{C_{t}-\beta y}{\sqrt{1-\beta^{2}}}\right) \phi(y)=\frac{1}{2 \pi} e^{y \rho \sigma \sqrt{T}-\left(\frac{C-\beta y}{\sqrt{1-\beta^{2}}}\right)^{2} / 2} e^{-y^{2} / 2}
$$

The power of the expression above can be expressed as

$$
-\frac{1}{2}\left[\left(\frac{y}{\sqrt{1-\beta^{2}}}\right)^{2}-2 y \rho \sigma \sqrt{T}-2 \frac{\beta C}{\sqrt{1-\beta^{2}}} \frac{y}{\sqrt{1-\beta^{2}}}+\frac{C^{2}}{1-\beta^{2}}\right]=-\frac{1}{2}\left[\left(\frac{y}{\sqrt{1-\beta^{2}}}\right)^{2}-2\left(\rho \sigma \sqrt{T} \sqrt{1-\beta^{2}}+\frac{\beta C}{\sqrt{1-\beta^{2}}}\right) \frac{y}{\sqrt{1-\beta^{2}}}+\frac{C^{2}}{1-\beta^{2}}\right]
$$

Denoting $A_{1}=\rho \sigma \sqrt{T} \sqrt{1-\beta^{2}}+A_{2}$ where $A_{2}=\frac{\beta C}{\sqrt{1-\beta^{2}}}$, the expression can be expressed as

$$
-\frac{1}{2}\left(\frac{y}{\sqrt{1-\beta^{2}}}-A_{1}\right)^{2}+\frac{1}{2}\left(A_{1}^{2}-\frac{C^{2}}{1-\beta^{2}}\right)
$$

The last term can be further simplified as

$$
A_{1}^{2}-\frac{C^{2}}{1-\beta^{2}}=\left(\rho \sigma \sqrt{T} \sqrt{1-\beta^{2}}+A_{2}\right)^{2}-\frac{C^{2}}{1-\beta^{2}}=\rho^{2} \sigma^{2} T\left(1-\beta^{2}\right)+2 A_{2} \rho \sigma \sqrt{T} \sqrt{1-\beta^{2}}+A_{2}^{2}-\frac{C^{2}}{1-\beta^{2}}
$$

Replacing $A_{2}=\frac{\beta C}{\sqrt{1-\beta^{2}}}$ in the expression above yields

$$
A_{1}^{2}-\frac{C^{2}}{1-\beta^{2}}=\rho^{2} \sigma^{2} T\left(1-\beta^{2}\right)+2 C \beta \rho \sigma \sqrt{T}+\frac{C^{2} \beta^{2}-C^{2}}{1-\beta^{2}}=-(C-\beta \rho \sigma \sqrt{T})^{2}+\rho^{2} \sigma^{2} T
$$

Denoting

$$
v=\left(\frac{y}{\sqrt{1-\beta^{2}}}-A_{1}\right)
$$

and

$$
A=C-\beta \rho \sigma \sqrt{T}
$$

the function $h(y)$ can be expressed as the function of $v$

$$
h(v)=\frac{1}{2 \pi} e^{-\frac{1}{2} v^{2}-\frac{1}{2} A^{2}+\frac{1}{2} \rho^{2} \sigma^{2} T}=\phi(v) \phi(A) e^{\frac{1}{2} \rho^{2} \sigma^{2} T}
$$

\section{References}

[1] Basel Committee on Banking Supervision (BCBS) (2010), 'Basel III: A global regulatory framework for more resilient banks and banking systems', Report, December, Bank for International Settlements, available at https://www.bis.org/publ/bcbs189.pdf

[2] Redon C. (2006), 'Wrong Way Risk Modelling', Risk, April issue, pp. 90-95.

[3] Brigo D. and Pallavicini A. (2006), 'Counterparty Risk and
Contingent CDS Valuation Under Correlation Between Interest-Rates and Default', SSRN Electronic Journal, August, available at DOI: $10.2139 /$ ssrn.926067.

[4] Brigo D., Chourdakis K. and Bakkar I. (2008) 'Counterparty Risk Valuation for Energy-Commodities Swaps', Fitch Solutions, available at https://arxiv.org/abs/0901.1099

[5] Pykhtin M. and Rosen D. (2010) 'Pricing Counterparty Risk at the Trade Level and Credit Valuation Adjustment Allocations', The Journal of Credit Risk, Vol. 6, No. 4, pp. 3-38. 
[6] Cespedes J., Herrero J., Rosen D. and Saunders D. (2010) 'Effective Modeling of Wrong Way Risk, Counterparty Credit Risk Capital, and Alpha in Basel II', The Journal of Risk Model Validation, Vol. 4, No. 1, pp. 71-98.

[7] Rosen D. and Saunders D. (2012) 'CVA the Wrong Way', Journal of Risk Management in Financial Institutions, Vol. 5, No. 3, pp. 252-272.

[8] Hull J. and White A. (2012) 'CVA and Wrong-Way Risk', Financial Analysts Journal, Vol. 68, No. 5, pp. 58-69.

[9] Ghamami S. and Goldberg L. (2014) 'Stochastic Intensity Models of Wrong Way Risk: Wrong Way CVA Need Not Exceed Independent CVA', The Journal of Derivatives, Vol. 21, No. 3, pp. 24-35.

[10] Lipton A. and Sepp A. (2009) 'Credit Value Adjustment for Credit Default Swaps via the Structural Default Model', The Journal of Credit Risk, Vol. 5, No. 2, pp. 123-146.

[11] Pang T., Chen W. and Li L. (2015) 'CVA Wrong Way Risk Multiplier Decomposition and Efficient CVA Curve', Journal of Risk Management in Financial Institutions, Vol. 8, No. 4, pp. 390-404.

[12] Chung T-K. and Gregory J. (2019) 'CVA Wrong-Way Risk: Calibration Using a Quanto CDS Basis', Risk. net, 02 July 2019.

[13] Pan K. (2018) 'CVA Pricing for Commodities with WWR', Cutting Edge Energy Risk, July issue 2018.

[14] Pan K. and Khandrika C. (2019) 'Credit Valuation Adjustment Wrong Way Risk in a Gaussian Copula Model', The Journal of Credit Risk, Vol. 15, No. 4, pp. 43-57.
[15] Gregory J. (2013) 'Counterparty Credit Risk and Credit Value Adjustment: A Continuing Challenge for Global Financial Markets', The Fourth Edition, Wiley, West Sussex, UK.

[16] Gregory J. (2015) 'The xVA Challenge', The Third Edition, Wiley, West Sussex, UK.

[17] Brigo D. and F. Mercurio (2006) 'Interest Rate Models Theory and Practice with Smile, Inflation and Credit', The second Ed., Springer.

[18] Board of Governors of the Federal Reserve System (2020) 'Capital Assessments and Stress Testing Information Collection Q\&As', September, available at https://www.federalreserve.gov/publications/files/fr-y14qas.pdf

[19] Li D. (2000) 'On Default Correlation: a Copula Function Approach', The Journal of Fixed Income, Vol. 9, No 4, pp. 43 54. available at https://doi.org/10.2469/faj.v68.n5.6

[20] Vasicek, O. (2002) 'The Distribution of Loan Portfolio Value', Risk, Vol. 15, No. 12, 160-162.

[21] O'Kane D. (2008) 'Modelling Single-name and Multi-name Credit Derivatives', Wiley, New Jersey.

[22] Pan K. (2017) 'An Analytical Expression for Bivariate Normal Distribution', SSRN Electronic Journal, February 2017, available at $h$ ttps://ssrn.com/abstract $=2924071$

[23] Vazza D. and Kraemer N. (2021) '2020 Annual Global Corporate Default and Rating Transition Study', RatingsDirect $S \& P, \quad$ April 7 2021, available at https://www.maalot.co.il/Publications/TS20210408160139.PDF 